Rev. Adm. Saúde (On-line), São Paulo, v. 20, n. 81: e254, out. - dez. 2020, Epub 24 dez. 2020 http://dx.doi.org/10.23973/ras.81.254

ARTIGO ORIGINAL

\title{
Atenção primária à saúde: avaliação de indicadores de estrutura e de processo em um município de pequeno porte
}

Primary health care: evaluation of structure and process indicators in a small municipality

\section{Alfredo Fittipaldi Massaro, Joéli Fernanda Basso², Izaltina Adão Alberto, José Niituma Ogata ${ }^{4}$}

1. Mestrando, Gestão para a Competitividade, Linha Saúde, Escola de Administração de Empresas, Fundação Getúlio Vargas (FGV), São Paulo SP.

2. Enfermeira. Doutora em enfermagem pelo Programa de Pós Graduação em Enfermagem da Universidade Federal de Santa Catarina. Florianópolis, Santa Catarina, Brasil

3. Doutoranda, Programa de Pós-Graduação em Saúde Coletiva, Centro de Ciências da Saúde, Universidade Federal de Santa Catarina (UFSC), Florianópolis SC.

4. Pesquisador Associado do Centro de Estudos em Planejamento e Gestão de Saúde da Escola de Administração de São Paulo da FGV, São Paulo SP.

\section{RESUMO}

Objetivo: Avaliar a experiência de gestão da Atenção Primária à Saúde (APS), a partir dos indicadores de estrutura e processo, de um município de pequeno porte do Estado de São Paulo, no período de 2012 a 2019. Método: Trata-se de um estudo quantitativo, observacional descritivo, longitudinal e retrospectivo, que se utilizou de dados coletados em bases públicas e documentos oficiais e em relatórios de gestão pública. Resultados: Os resultados demonstram que, com a implantação das equipes de Estratégia da Saúde da Família (ESF) a partir de 2013 e com sua ampliação para a capacidade máxima (em 100\%) 
entre os anos de 2014 e 2015, propiciou-se maior acesso aos usuários com melhoria dos indicadores de processo, inclusive no controle das condições sensíveis à atenção primária. Após 2016 até o ano de 2019 com a exclusão de cinco equipes de ESF, as oportunidades de acesso foram reduzidas, assim como o número de atendimentos e as atividades por elas desenvolvidas, comprometendo atributo da longitudinalidade do cuidado em saúde.

Palavras-chave: Avaliação em atenção à saúde. Atenção primária à Saúde. Gestão em Saúde. Saúde Pública. Sistema Único de Saúde.

\section{ABSTRACT}

Objective: To evaluate the management experience of Primary Health Care $(P H C)$, based on the indicators of structure and process, of a small municipality in the State of São Paulo, in the period from 2012 to 2019. Method: This is a quantitative, observational, descriptive, longitudinal, and retrospective study, which used data collected in public databases and official documents and in public service reports. Results: The results demonstrate that, with the implementation of the Family Health Strategy (ESF) teams from 2013 and with their expansion to maximum capacity (by 100\%) between the years 2014 and 2015, greater access was provided to users with improved process indicators, including the control of conditions sensitive to primary care. After 2016 until 2019 with the exclusion of five FHS teams, the opportunities for access were reduced, as well as the number of visits and the activities performed by them, compromising the longitudinality attribute of health care.

Keywords: Health care evaluation. Primary Health Care. Health Management. Public Health, Unified Health System.

\section{INTRODUÇÃO}

A Atenção Primária à Saúde é considerada a porta de entrada do SUS e orientativa de todo o fluxo dos serviços, dos mais simples aos mais complexos, nas redes de saúde. Para que os objetivos individuais e coletivos em saúde sejam atingidos, os gestores precisam assegurar infraestrutura e planejar e executar ações orientadas pelos princípios da universalidade, da acessibilidade, da continuidade do cuidado, da integralidade da atenção, da responsabilização, da humanização e da equidade. Se espera que considerem a promoção e a proteção da saúde, a prevenção de agravos, o diagnóstico, o tratamento, a reabilitação, a redução de danos e a manutenção da saúde das coletividades. 
Tais ações ainda devem ser orientadas por alguns princípios da gestão pública, dos quais destacam-se a transparência em prestar contas à sociedade, a liderança, o direcionamento das ações previstas nas políticas de saúde e a capacidade de produtividade necessária para atender de forma individual ou coletiva os cidadãos, em prol do bem-estar da população ${ }^{1}$.

E planejar e operacionalizar a atenção à saúde de uma população adstrita tornando-a qualitativa e quantitativamente mensurável, se configura em um grande desafio. Para superá-lo, a estratégia dos gestores do SUS têm sido promover a evolução do Programa Estratégia de Saúde da Família, para maior oferta de serviços essenciais de saúde à população. Para Mendes ${ }^{2}$, em linhas gerais, o SUS vem alcançando seus objetivos, mas mantém fragilidades que prejudicam a implementação de propostas estruturantes que favoreçam a expansão ao acesso da população às unidades básicas de saúde, a descentralização dos serviços de atenção à saúde, aos processos de regionalização nos Estados e seus pactos com os municípios, assim como a coordenação dos cuidados e a saúde de forma integral. Existe então urgência no fortalecimento da APS e clareza das suas interfaces com toda a rede, utilizando recursos com eficiência e transparência, e garantindo efetividade e qualidade nos serviços prestados ${ }^{3}$.

E falar em gestão e melhorias exige que se fale de métodos de avaliação de serviços, demanda de atendimento e qualidade em serviços de saúde. Donabedian ${ }^{4}$ nos permite esse diálogo a partir de um sistema de avaliação periódica, voluntária e reservada. Para o autor, os indicadores de qualidade capazes de avaliar os serviços de saúde se agrupam em três categorias: Estrutura, Processo e Resultado. Em estrutura são avaliados os atributos do local onde o serviço de saúde é prestado, nos quais incluem-se os recursos materiais (instalações, equipamentos e o investimento da estrutura), os recursos humanos (quantidade dos profissionais), o recurso da estrutura organizacional (profissionais da saúde) e os recursos financeiros (mecanismos de financiamento de investimento nas estruturas físicas e na capacitação dos profissionais). Já em processo, deverão ser avaliadas as atividades realizadas pelos profissionais para com os cuidados à saúde dos usuários, os quais são classificados como cuidado técnico (conhecimento tecnológico e científico da medicina e áreas do setor da saúde) e a relação entre o profissional e o paciente durante o tempo em que o usuário receberá o atendimento, desde o diagnóstico até o tratamento, assim como dar continuidade e acompanhamento durante a vida do usuário. E em resultado, devem ser avaliados os efeitos que foram produzidos durante os cuidados prestados pelos profissionais de saúde, a satisfação do usuário e do profissional, ou seja, o estado de saúde do paciente diante dos cuidados promovidos e recebidos durante e após o atendimento.

Essas três dimensões (estrutura, processo e resultado) mantém relação estreita, mas nem sempre causal, ou seja, uma boa estrutura não significa ter bons resultados, mas bons resultados são muito mais fáceis de se conseguir com uma estrutura adequada, profissionais bem capacitados e uma relação de confiança e troca entre pacientes e equipe. Para Donabedian ${ }^{5}$ esta abordagem de três dimensões para avaliação da qualidade em serviços de saúde é possível porque boa estrutura aumenta a probabilidade de bom processo, e 
bom processo aumenta a probabilidade de obter melhorias na saúde e bemestar dos indivíduos ou populações, ou seja, bom resultado. Desta forma, é possível entender que, condições estruturais podem ser tanto desfavoráveis quanto condutivas ao bom cuidado, não sendo possível afirmar se o cuidado, em função dessas, será bom ou ruim.

Considerando-se que os indicadores de resultado, particularmente no que se refere aos desfechos clínicos, estão fortemente relacionados à atenção secundária e terciária, principalmente no atendimento hospitalar, os quais sofrem influência de vários fatores relacionados à estrutura de gestão do SUS, sobre os quais a APS em um município de pequeno porte, frequentemente, não tem governança, este estudo se propõem a avaliar a experiência de gestão da Atenção Primária à Saúde (APS), limitada aos indicadores de estrutura e processo, de um município de pequeno porte do Estado de São Paulo, no período de 2012 a 2019.

É importante ressaltar que os indicadores são ferramentas que irão apoiar a gestão na tomada de decisão, além de auxiliar na construção de diretrizes, oportunizando melhorias nas ações de saúde que qualifiquem cada vez mais o atendimento e ampliem o acesso dos usuários do SUS aos serviços de saúde.

\section{METODOLOGIA}

Com o objetivo de avaliar indicadores de estrutura e de processo na atenção primária à saúde em Charqueada, um município de pequeno porte localizado no interior do estado de São Paulo, possui uma área territorial de 175,8 km² e população estimada de 17.190 habitantes ${ }^{6}$ adotou-se uma pesquisa observacional descritiva, longitudinal e retrospectiva, de abordagem quantitativa.

O estudo baseou-se em dados secundários, obtidos a partir de bases públicas consolidadas, documentos oficiais, relatórios de gestão pública do sítio eletrônico do Departamento de Informática do SUS (DataSus, e-gestor, CNES), que pertence ao Ministério da Saúde, além do Sistema de Informações sobre Orçamentos Públicos em Saúde (SIOPS) e a base de dados do Sistema Estadual de Análise de Dados (Seade), que pertence ao governo do estado de São Paulo e está vinculado à Regional de Saúde (DRS-10/Piracicaba), a qual pertence o município de Charqueada/SP. Realizou-se análise dos dados destes indicadores no período de 2012 a 2019.

Foram considerados para avaliação os pressupostos de Donabedian ${ }^{6}$ sobre indicadores de estrutura e processo. Já os indicadores de resultado foram desconsiderados neste estudo por tratar-se basicamente dos desfechos clínicos, normalmente relacionados à atenção secundária e terciária, principalmente no atendimento hospitalar, os quais sofrem influência de vários fatores relacionados à estrutura de gestão do SUS, sobre os quais a APS em um município de pequeno porte, frequentemente, não tem governança. Além de necessitarem de tempo de observação para serem concluídos, sendo considerados mais complexos na sua mensuração. 
Logo, a partir da abordagem quantitativa foram analisados os seguintes indicadores de estrutura: cobertura de equipes de ESF e número de consultas médicas anuais. E os seguintes indicadores de processo ligados à APS: quantidade de diagnósticos sensíveis a $A B$ e internação hospitalar, número de atendimentos a gestantes. Esses dados foram obtidos por e-mail junto à Diretoria Regional de Saúde (DIR-XV/Piracicaba).

Além disso, por se tratar de um estudo com dados de oito anos de série histórica, o estudo tem característica longitudinal, sendo esse um dos atributos da APS que favorece o engajamento do paciente, com a mesma equipe realizando $o$ atendimento ao longo do tempo.

O presente estudo foi submetido ao Comitê de Conformidade Ética em Pesquisas Envolvendo Seres Humanos da Fundação Getúlio Vargas, tendo sido autorizada a realização da pesquisa por envolver apenas dados secundários e anônimos.

\section{RESULTADOS}

\section{Indicadores de estrutura}

\section{Cobertura}

A avaliação no período delimitado, entre 2012 e 2019 no município de Charqueada, aponta que ao longo do período passou pela experiência de sair de zero de cobertura de ESF no ano de 2012, chegando a $100 \%$ de cobertura no ano de 2014 e, em seguida, regredindo no percentual de cobertura até 2019. Na evolução da cobertura de atendimento da APS/AB, além da cobertura do atendimento à população com as equipes de ESF no período estudado, é possível verificar que o crescimento da cobertura acompanha o esforço de implantação das equipes de Saúde da Família e, após período de cobertura em $100 \%$, regride com a diminuição das equipes. Os números indicam o impacto positivo na cobertura, ou seja, a maior disponibilidade de serviços e, consequente, maior acesso para a população de Charqueada/SP. Com isso, é possível inferir que no ano de 2016, com a dissolução das equipes de ESF a estratégia não refletiu manutenção dos índices de cobertura, chegando a menos de 50\% em 2018, o segundo pior índice de todo o período estudado (Tabela 1). 
Tabela 1 - Cobertura APS/AB/ESF e número de equipes de ESF 2012/2019

\begin{tabular}{lcccccccc}
\hline & $\mathbf{2 0 1 2}$ & $\mathbf{2 0 1 3}$ & $\mathbf{2 0 1 4}$ & $\mathbf{2 0 1 5}$ & $\mathbf{2 0 1 6}$ & $\mathbf{2 0 1 7}$ & $\mathbf{2 0 1 8}$ & $\mathbf{2 0 1 9}$ \\
\hline $\begin{array}{l}\text { COBERTURA } \\
\text { APS/AB }\end{array}$ & $40,35 \%$ & $68,79 \%$ & $100 \%$ & $100 \%$ & $100 \%$ & $65,93 \%$ & $47,40 \%$ & $51,69 \%$ \\
$\begin{array}{l}\text { COBERTURA } \\
\text { ESF }\end{array}$ & $0 \%$ & $22,42 \%$ & $100 \%$ & $100 \%$ & $22,99 \%$ & $20,77 \%$ & $20,57 \%$ & $20,28 \%$ \\
$\begin{array}{l}\text { NÚMERO DE } \\
\text { EQUIPES ESF }\end{array}$ & 0 & 1 & 6 & 5 & 1 & 1 & 1 & 1 \\
\end{tabular}

Fonte: Ministério da Saúde/ Sistema de Informações Ambulatoriais (SAI/SUS) / Adaptado pelos autores

A Tabela 2 demonstra o número de unidades de Atenção Básica, de 2012 a 2019, indicando uma nova unidade a partir do ano de 2013.

Tabela 2 - Número de unidade de Saúde (AB)

\begin{tabular}{lcccccccc}
\hline & 2012 & 2013 & 2014 & 2015 & 2016 & 2017 & 2018 & 2019 \\
\hline TOTAL & 4 & 5 & 5 & 5 & 5 & 5 & 5 & 5
\end{tabular}

Fonte: Ministério da Saúde/Sistema de Informações Ambulatoriais (SAI/SUS) / Adaptado pelos autores

\section{Consultas e procedimentos ambulatoriais}

O número de consultas e procedimentos ambulatoriais realizados de 2012 a 2019 por médicos, enfermeiros e técnicos e/ou auxiliares de enfermagem indica algum problema de registro de produção no ano de 2012, não havendo o registro de implantação de equipes de ESF, conforme representado na Tabela 3. A partir de 2013, com a implantação das equipes de ESF, são realizados os registros, sendo possível identificar um grande acréscimo na produção até 0 ano de 2016. Já em 2017, 2018 e 2019, o atendimento médico é reduzido a menos da metade quando comparado ao ano de 2016. O atendimento de enfermeiros e dos técnicos/auxiliares de enfermagem também sofrem queda. É possível verificar que a produção dos profissionais atinge picos de 28.883 e 28.894 em 2015 e 2016 respectivamente, mas em seguida regridem $46 \%$ em 2017 , tendência que também é observada na cobertura de atendimento à população, caracterizando a redução de acesso. 
Tabela 3- Número de consultas/ambulatoriais de 2012 a 2019 (ESF)

\begin{tabular}{lllllllll}
\hline Procedimento & $\mathbf{2 0 1 2}$ & $\mathbf{2 0 1 3}$ & $\mathbf{2 0 1 4}$ & $\mathbf{2 0 1 5}$ & $\mathbf{2 0 1 6}$ & $\mathbf{2 0 1 7}$ & $\mathbf{2 0 1 8}$ & $\mathbf{2 0 1 9}$ \\
\hline MÉDICO & 0 & 0 & 3.125 & 6.568 & 4.882 & 2.060 & 2.086 & 2.425 \\
ENFERMEIROS & 0 & 29 & 1.387 & 3.027 & 2.780 & 1.549 & 315 & 1.450 \\
$\begin{array}{l}\text { TÉCNICO/ } \\
\begin{array}{l}\text { AUXILIAR DE } \\
\text { ENFERMAGEM }\end{array}\end{array}$ & 0 & 1.012 & 13.688 & 19.238 & 21.232 & 12.020 & 19.396 & 8.178 \\
Total & $\mathbf{0}$ & $\mathbf{1 . 0 4 1}$ & $\mathbf{1 8 . 2 0 0}$ & $\mathbf{2 8 . 8 8 3}$ & $\mathbf{2 8 . 8 9 4}$ & $\mathbf{1 5 . 6 2 9}$ & $\mathbf{2 1 . 7 9 4}$ & $\mathbf{1 2 . 0 5 3}$ \\
\hline
\end{tabular}

Fonte: Ministério da Saúde/ Sistema de Informações Ambulatoriais/ Adaptado pelos autores

\section{Indicadores de processo}

\section{Número de profissionais das equipes ESF}

De acordo com a Tabela 4 nota-se que no ano de 2012 não havia equipe ESF (Estratégia Saúde da Família). Já nos anos de 2013 a 2016 foram registradas equipes, sendo possível identificar o aumento no número de profissionais, com característica adequada às exigências do programa Saúde da Família, ou seja, equipes multidisciplinares com médico, enfermeiro, técnico ou auxiliar de enfermagem e agentes comunitários. No entanto, observa-se alterações significativas a partir do ano de 2016.

Tabela 4 - Número de profissionais/unidades de 2012/2019 (ESF)

\begin{tabular}{|c|c|c|c|c|c|c|c|c|}
\hline & 2012 & 2013 & 2014 & 2015 & 2016 & 2017 & 2018 & 2019 \\
\hline MÉDICO & 0 & 0 & 5 & 5 & 5 & 1 & 0 & 0 \\
\hline EMFERMEIRO & 0 & 1 & 6 & 4 & 4 & 1 & 1 & 1 \\
\hline TÉCNICOS DE & & & & & & & & \\
\hline $\begin{array}{l}\text { ENFERMAGEM/AUXILIAR } \\
\text { DE ENFERMAGEM }\end{array}$ & 0 & 1 & 5 & 5 & 5 & 1 & 1 & 1 \\
\hline $\begin{array}{l}\text { AGENTES } \\
\text { COMUNITÁRIOS DE } \\
\text { SAÚDE }\end{array}$ & 0 & 6 & 33 & 31 & 6 & 6 & 5 & 4 \\
\hline Total & $\mathbf{0}$ & 8 & 49 & 45 & 20 & 19 & 7 & 6 \\
\hline
\end{tabular}

Fonte: Ministério da Saúde/Sistema de Informações Ambulatoriais (SAI/SUS) / Adaptado pelos autores

Na Tabela 5 é apresentado o número de cadastros realizados pelos Agentes Comunitários de Saúde (ACS) de 2012 a 2019, sendo possível evidenciar o volume de cadastros concentrados entre os anos de 2013 a 2016, com destaque significativo no ano de 2014. 
Tabela 5-Cadastros domiciliar/famílias realizados pelos ACS/ESF

\begin{tabular}{lcrrrrrrc}
\hline & $\mathbf{2 0 1 2}$ & $\mathbf{2 0 1 3}$ & $\mathbf{2 0 1 4}$ & $\mathbf{2 0 1 5}$ & $\mathbf{2 0 1 6}$ & $\mathbf{2 0 1 7}$ & $\mathbf{2 0 1 8}$ & $\mathbf{2 0 1 9}$ \\
\hline Cadastros & 0 & 419 & 2.995 & 356 & 228 & 0 & 3 & 0 \\
\hline
\end{tabular}

Fonte: Sisab/ Adaptado pelos autores

Já Tabela 6 é possível observar os cadastros individuais, realizados pelos mesmos agentes, no mesmo período da Tabela 5, evidenciando da mesma forma, a concentração de cadastros realizados entre os anos de 2013 a 2016.

Tabela 6- Cadastros individuais realizados pelos ACS/ESF

\begin{tabular}{lllllllll}
\hline & $\mathbf{2 0 1 2}$ & $\mathbf{2 0 1 3}$ & $\mathbf{2 0 1 4}$ & $\mathbf{2 0 1 5}$ & $\mathbf{2 0 1 6}$ & $\mathbf{2 0 1 7}$ & $\mathbf{2 0 1 8}$ & $\mathbf{2 0 1 9}$ \\
\hline Cadastros & 0 & 1.470 & 8.773 & 1.205 & 975 & 2 & 6 & 0 \\
\hline
\end{tabular}

Fonte: Sisab/ Adaptado pelos autores

Na Tabela 7 é apresentada a evolução das visitas domiciliares realizadas pelos Agentes Comunitários de Saúde no período dos oito anos desse estudo.

Tabela 7- Visitas domiciliares realizadas pelos ACS/ESF

\begin{tabular}{lllllllll}
\hline & $\mathbf{2 0 1 2}$ & $\mathbf{2 0 1 3}$ & $\mathbf{2 0 1 4}$ & $\mathbf{2 0 1 5}$ & $\mathbf{2 0 1 6}$ & $\mathbf{2 0 1 7}$ & $\mathbf{2 0 1 8}$ & $\mathbf{2 0 1 9}$ \\
\hline Visitas & 0 & 585 & 15.342 & 31.027 & 20.697 & 4.063 & 3.298 & 1.596 \\
\hline
\end{tabular}

Fonte: Sisab/ Adaptado pelos autores

\section{Quantidade de gestantes atendidas pelas ESF}

A Tabela 8 apresenta os atendimentos à gestante, no período de 2012 a 2019, divididos em puericultura, consulta pré-natal e consulta puerperal, evidenciando maiores índices entre 2013 e 2016, seguida de sensível redução no número de atendimento pré-natal a partir de 2017. A mesma tendência se repete no item consulta puerperal, cujos maiores índices de atendimento ocorreram nos anos de 2013, 2014, 2015. e 2016. 
Tabela 8 - Número de consultas/ gestantes de 2012 a 2019

\begin{tabular}{lllllllll}
\hline Procedimento & $\mathbf{2 0 1 2}$ & $\mathbf{2 0 1 3}$ & $\mathbf{2 0 1 4}$ & $\mathbf{2 0 1 5}$ & $\mathbf{2 0 1 6}$ & $\mathbf{2 0 1 7}$ & $\mathbf{2 0 1 8}$ & $\mathbf{2 0 1 9}$ \\
\hline PUERICULTURA $\left(^{*}\right)$ & - & 9 & 73 & 33 & 47 & 34 & 24 & 16 \\
$\begin{array}{l}\text { CONSULTA PRE- } \\
\text { NATAL }\end{array}$ & 1.159 & 2.657 & 2.130 & 2.210 & 1.791 & 1.244 & 1.150 & 1.169 \\
$\begin{array}{l}\text { CONSULTA } \\
\text { PUERPERAL }\end{array}$ & 142 & 180 & 202 & 217 & 160 & 130 & 165 & 117 \\
Total & $\mathbf{1 . 3 0 1}$ & $\mathbf{2 . 8 4 6}$ & $\mathbf{2 . 4 0 5}$ & $\mathbf{2 . 4 6 0}$ & $\mathbf{1 . 9 9 8}$ & $\mathbf{1 . 4 0 8}$ & $\mathbf{1 . 3 3 9}$ & $\mathbf{1 . 3 0 2}$ \\
\hline
\end{tabular}

Fonte: Ministério da Saúde/ Sistema de Informações Ambulatoriais (SAI/SUS) / Adaptado pelos autores

(*) Consulta para acompanhamento de crescimento e desenvolvimento

\section{Diagnósticos e condições sensíveis à APS}

$\mathrm{Na}$ Tabela 9 estão apresentados os números totais de diagnósticos/condições sensíveis à APS, que por sua vez, se relacionam aos dados de internações, já que o maior número de diagnósticos deve refletir em números menores nas internações.

Tabela 9- Diagnóstico de doenças sensíveis a AB3/APS

\begin{tabular}{lcccccccc}
\hline & $\mathbf{2 0 1 2}$ & $\mathbf{2 0 1 3}$ & $\mathbf{2 0 1 4}$ & $\mathbf{2 0 1 5}$ & $\mathbf{2 0 1 6}$ & $\mathbf{2 0 1 7}$ & $\mathbf{2 0 1 8}$ & $\mathbf{2 0 1 9}$ \\
\hline $\begin{array}{l}\text { Quantidades de } \\
\text { diagnósticos/doenças } \\
\text { sensíveis a Ab3 - APS }\end{array}$ & 203 & 173 & 141 & 125 & 124 & 122 & 152 & 100 \\
\hline
\end{tabular}

Fonte: Ministério da Saúde/Sistema de Informações Ambulatoriais (SAI/SUS) / Adaptado pelos autores

Na Tabela 10 são apresentados os montantes de produção ambulatorial, com um crescimento entre 2013 e 2016, seguindo tendência de retração em 2017, 2018 e 2019, mas ainda assim acima do total de 2012, menor número registrado no período de estudo.

Tabela 10- Produção ambulatorial de exames do SUS

\begin{tabular}{lllllllll}
\hline & $\mathbf{2 0 1 2}$ & $\mathbf{2 0 1 3}$ & $\mathbf{2 0 1 4}$ & $\mathbf{2 0 1 5}$ & $\mathbf{2 0 1 6}$ & $\mathbf{2 0 1 7}$ & $\mathbf{2 0 1 8}$ & $\mathbf{2 0 1 9}$ \\
\hline Exames & 27.818 & 56.325 & 57.897 & 47.948 & 65.414 & 46.565 & 41.235 & 46.356 \\
\hline
\end{tabular}

Fonte: Ministério da Saúde/Sistema de Informações Ambulatoriais (SAI/SUS) / Adaptado pelos autores 
Destaca-se que a produção ambulatorial, considerando o volume de exames realizados anualmente, permite inferir que mais diagnósticos puderam ser realizados, caracterizando mais resolubilidade, o que no contexto do SUS significa maior capacidade das equipes em reconhecer as necessidades locais de saúde e ofertar ações para atendimento das mesmas.

\section{DISCUSSÃO}

Os resultados da atuação das equipes de ESF apresentados neste artigo indicam que é possível aumentar o acesso aos cuidados de saúde e fortalecer a APS com a utilização desse formato organizacional. Por meio da avaliação dos indicadores de estrutura e de processo podemos observar que no período delimitado entre os anos de 2012 e 2019 houve grande oscilação no acesso e nas atividades desenvolvidas pelas equipes.

O período delimitado neste estudo compreende uma série histórica com dados relevantes por englobar alterações significativas na estratégia de atuação da gestão municipal de saúde de Charqueada/SP. No ano de 2012, início do estudo, a cidade optava por uma política de Atenção Básica sem utilizar as equipes de ESF e sem a devida atenção aos atributos da APS: atenção ao primeiro acesso, a longitudinalidade, a integralidade e a coordenação dos cuidados. A partir de $2013 \mathrm{com}$ a implantação da primeira equipe e consequentemente de sua plena capacidade até o ano de 2015 foi possível ver o aumento nos números de atendimentos, a ação das equipes e especialmente dos ACS com a comunidade e melhora significativa do acesso proporcionado aos usuários locais.

Na Tabela 3 é possível verificar que sem as equipes de ESF no ano de 2012, a cobertura da Atenção Básica oscilou entre 0\% a 40,35\%, mas com a implantação das seis equipes de ESF a partir de 2014 chegou a $100 \%$ de cobertura em dois anos, o que indica um aumento significativo no acesso aos serviços de saúde de APS. Contudo, a partir de 2016 a cobertura regride com a redução das equipes. No mesmo período, conforme demonstra Tabela 5, o cadastro de famílias e de indivíduos (Tabelas 5 e 6) se concentrou entre 2013 e 2016, período de manutenção das ESF.

Em consequência à estruturação das equipes, a Tabela 8 demonstra crescimento na produção das equipes de 2013 a 2016, provavelmente resultado de uma política de mais acesso e relacionamento com os usuários do SUS. Em 2017, 2018 e 2019, o atendimento médico é reduzido a menos da metade do que em 2016, o atendimento realizado pela equipe de enfermagem também sofre queda e o de técnico/auxiliar de enfermagem e apesar da redução mantém níveis mais elevados.

Num recorte da análise anterior, verifica-se que a produção dos profissionais atinge picos de 28.883 e 28.894 em 2015 e 2016, mas em seguida regridem $46 \%$ em 2017, tendência que também é observada na cobertura de atendimento à população, ou seja, redução de acesso. 
No atendimento à gestante, apontado na Tabela 8, houve crescimento dos atendimentos em puericultura, consulta pré-natal e consulta puerperal no período de 2013 a 2016, mesmo período de atuação da cobertura total das equipes de ESF, o que demonstra maior adesão da gestante ao acompanhamento pré-natal.

Comparando-se a média de diagnósticos de diagnósticos/condições sensíveis à APS (Tabela 09) entre 2012/2016, que foi de 160,6 diagnósticos por ano, e o período de 2016/2019 que foi de 124,5, nota-se redução na eficácia do sistema de APS.

Um fato relevante nos números apresentados diz respeito ao trabalho dos Agentes Comunitários de Saúde (ACS), que são responsáveis pela manutenção do contato e acompanhamento da comunidade atendida pela unidade de ESF. Cabe a esse profissional a visitação periódica e acompanhamento do paciente, além de orientação com relação à dispensa de medicamentos, agendamento de consultas e a atualização cadastral dos indivíduos e seus domicílios.

De 2013 a 2016, os ACS formavam o grande contingente de trabalho das unidades, iniciando com 6 colaboradores em 2013 e chegando a 33 profissionais em 2014, mas o número total regrediu para 6, 5 e 4, respectivamente entre 2017, 2018 e 2019. Com esta regressão das equipes de ESF e consequentemente dos ACS verifica-se a queda acentuada da atualização dos registros nos cadastros dos indivíduos e dos domicílios por eles visitados.

Outro dado relevante relacionado à equipe é a produção do atendimento de técnicos e auxiliares de enfermagem no período do estudo, que significa parcela muito representativa no total dos atendimentos. Os dados indicam que boa parcela da demanda das unidades é de cuidados básicos, com resolução rápida e/ou baixo nível de complexidade médica.

Os números indicam que é possível manter a comunidade vinculada à unidade com o trabalho de base de agentes e técnicos e auxiliares evitando agravamentos de doenças e abandono de tratamento. É óbvio que o papel dos outros profissionais é indispensável, mas a ponta do atendimento deve oferecer retaguarda à comunidade para ser possível aumentar o impacto das equipes de ESF.

As ações de prevenção e educação para a saúde, uma das frentes da APS, pode contar com o apoio desses técnicos, que mantém relação estreita com a comunidade e conhecem o histórico das famílias e indivíduos, alguns desde o pré-natal.

Apesar dos resultados em termos quantitativos terem sido expressivos no período de atuação da ESF, o número de unidades físicas existentes se manteve entre 2013 e 2019 (Tabela 2), o que indica possibilidade de atendimento da população sem investimentos em construção de mais unidades. 
Verifica-se pelos dados da Tabela 10 tendência bem semelhante aos outros resultados obtidos com os dados, com concentração de produção entre os anos de 2013 a 2016, quando alguma alteração no método reduz o número de atendimento para menos da metade dos anos anteriores, sendo 2017 o pior ano de resultado com 9.990 atendimentos, o que equivale ao segundo pior desempenho do período, mesmo assim é 35\% menor que o segundo pior desempenho.

Diante dos dados apresentados neste estudo é possível notar que a ação das equipes de ESF é uma estratégia positiva em comunidades pequenas com relação aos resultados totais.

Na esfera da análise quantitativa as mudanças implantadas de 2016 para 2017 não se mostraram eficazes, e percebe-se o abandono da proposta das ESF como estratégia principal de cobertura da população, além de redução, muitas vezes drásticas nos procedimentos, ações e resultados da Atenção Básica no município de Charqueada/SP.

Pode acrescentar-se ainda que gestão da Atenção Primária à Saúde necessita cada vez mais de informações, dados e indicadores que auxiliem seus gestores nas tomadas de decisões e para o aprimoramento dos processos, na estruturação das equipes e na capacitação dos profissionais. Fatores esses, determinantes para melhorar o atendimento dos usuários e fortalecer a APS.

\section{CONCLUSÃO}

Diante dos dados apresentados, pode-se concluir que os resultados obtidos neste estudo demonstram que, com a implantação das equipes de ESF, a partir de 2013, a população de Charqueada-SP teve mais acesso à atenção primária à saúde.

Por fim, fica evidente a importância da gestão das equipes de ESF de forma coordenada e com estrutura adequada. As equipes multiprofissionais, com coordenação do cuidado, propiciam o vínculo dos usuários, conseguindo inclusive um melhor controle das condições crônicas sensíveis à atenção primária. A avaliação e o acompanhamento dos indicadores, conforme proposto por Donabedian ${ }^{5}$ propicia a adoção de medidas corretivas, na estrutura e processos, buscando atingir os desfechos desejados. Logo, as diretrizes que serão realizadas pela governança poderão ser cada vez mais adequadas e melhor direcionadas às equipes, além disso, a execução dos serviços que serão realizadas pelo gestor e as equipes, serão mais qualificadas e as tomadas de decisões mais assertivas.

Sugere-se a realização de levantamentos relacionados à multiplicidade de variáveis, como a situação socioeconômica, região demográfica, serviços ofertados na saúde suplementar, entre outros para se mensurar, posteriormente, os indicadores de resultado. 
Como contribuições da pesquisa, aponta-se a possibilidade de reflexão sobre desafios e defasagens da gestão dos serviços em Atenção Primária à Saúde (APS), propondo sugestões na continuidade dos atendimentos e manutenção das equipes de ESF.

\section{REFERÊNCIAS}

1. Starfield B. Atenção primária: equilíbrio entre necessidades de saúde, serviços e tecnologia/ Bárbara Starfield. Brasília: UNESCO, Ministério da Saúde; 2002.

2. Brasil. Tribunal de Contas da União; Guia de Governança e Gestão em Saúde: aplicável a secretarias e conselhos de saúde: TCU, Secretaria de Controle Externo da Saúde, Brasília; 2018.

3. Mendes EV. Desafios do SUS. Brasília, DF: CONASS; 2019.

4. Ribeiro LA, Scatena JH. A avaliação da Atenção Primária à Saúde no contexto brasileiro: uma análise da produção científica entre 2007 e 2017. Saúde soc. Abr./jun. 2019; 28(2).

https://www.scielo.br/pdf/sausoc/v28n2/1984-0470-sausoc-28-02-95.pdf

5. Donabedian A. The quality of care: how can it be assessed? Journal of American Medical Association. Sep. 1988; 260(12):1743-8.

6. Donabedian A. The Definition of Quality and Approaches to its Assessment. In: Explorations in Quality Assessment and Monitoring, v. I. Ann Arbor, Michigan: Health Administration Press; 1988.

7. IBGE. Instituto Brasileiro de Geografia e Estatística. Charqueada. Disponível em: https://cidades.ibge.gov.br/brasil/sp/charqueada/panorama Acesso em: maio 15, 2020.

Recebido: 10 de novembro de 2020. Aceito: 24 de dezembro de 2020

Correspondência: Alberto José Niituma Ogata. E-mail: alberto.ogata@fgv.br 
Conflito de Interesses: os autores declararam não haver conflito de interesses.

(C) This is an Open Access article distributed under the terms of the Creative Commons Attribution License, which permits unrestricted use, distribution, and reproduction in any medium, provided the original work is properly cited 